\title{
Antisense DNA and RNA: progress and prospects
}

Using antisense DNA or RNA fragments to block the expression of selected genes, and thereby assess their function, is a powerful new tool for the molecular biologist. It is an approach that promises to be particularly useful in higher eukaryotes, where the genetic tools applicable to yeast and bacteria are not available. There are also obvious therapeutic implications in the use of antisense inhibition to block the expression of targeted viral genes or oncogenes. Last December a conference at the Banbury Center of Cold Spring Harbor Laboratory explored these aspects of antisense RNA and DNA.

A number of years before antisense RNA became fashionable, Paul Zamecnik and Mary Stephenson demonstrated the feasibility of using short antisense oligodeoxynucleotides to block the expression of targeted genes within intact cells (Zamecnik and Stephenson 1978). This work provided the first evidence that oligonucleotides, although highly negatively charged, could be transported into cells at some finite rate. John Goodchild (Worcester Foundation) described electron microscopy studies using radiolabeled derivatives that provided further evidence for intracellular uptake of oligonucleotides. With added evidence from other laboratories (Holt et al. 1988; Wickstrom et al. 1988), it is clear that oligonucleotides can be taken up into mammalian cells and can exert inhibitory effects on selected genes. The generality of this phenomenon and the mechanism of transport, however, remain uncertain.

To facilitate intracellular transport of oligonucleotides, Paul Miller and Paul T'so and their colleagues investigated analogs in which the negatively charged phosphate groups of the DNA strand are replaced by neutral methylphosphonate links. Intracellular transport of these derivatives is enhanced, but their intrinsic activity is far less than that of unmodified oligonucleotides. The concentration of oligonucleotide methylphosphonates required to inhibit protein synthesis in vitro is generally between 100 and $400 \mu \mathrm{M}$, compared with 0.5-5 $\mu \mathrm{M}$ for the corresponding unmodified sequences. The important difference is that oligonucleotide methylphosphonates do not form substrates for RNase $\mathrm{H}$ (J. Walder and R. Walder, University of Iowa). To dissect out the contribution of RNase $\mathrm{H}$, poly(rA)-oligo(dT) was used to block the activity of the enzyme present in reticulocyte lysates. This approach demonstrated that the arrest of translation by oligodeoxynucleotides which hybridize within the coding region or over the initiation codon is dependent on cleavage of the targeted mRNA by RNase $\mathrm{H}$. In some cases, but not all, binding of antisense sequences to the $5^{\prime}$ end of mRNAs was found to inhibit protein synthesis directly, presumably by interfering with the initiation of translation /see also, Lawson et al. 1986).

Miller and co-workers (Johns Hopkins University
School of Medicine), to enhance the activity of oligonucleotide methylphosphonates, have prepared derivatives that are further modified with psoralen. Irradiation with 365-nm light results in irreversible cross-linking of the oligonucleotide to the target sequence. Such derivatives block protein synthesis very effectively-in in vitro translation systems inhibition occurs in the micromolar concentration range. Claude Helene (INSERM) described a similar approach in which iron and copper chelate complexes were covalently attached to oligonucleotides. In the presence of reducing agents and oxygen, these complexes generate hydroxyl radicals that cleave the complementary strand to which the oligonucleotide is hybridized. This approach has many interesting applications in vitro. Its use in vivo, though, requires a mechanism to activate the system only after hybridization of the oligonucleotide to the targeted message.

Helene also described studies of a new class of oligonucleotides built from nucleosides having the $\alpha$-anomeric configuration rather than the naturally occurring $\beta$-form. These oligonucleotides hybridize strongly with complementary sequences. Strands of $\alpha-\beta$ hybrids run parallel, in contrast to the antiparallel orientation of $\beta-\beta$ duplexes. The $\alpha$-anomers are highly resistant to nuclease degradation. However, they do not form substrates with mRNAs that are recognized by RNase $\mathrm{H}$ and, therefore, are much less effective inhibitors than normal oligonucleotides.

Degradation of oligonucleotides in mammalian cells and in blood occurs most rapidly by exonucleases that can be blocked by end-group modifications (Walder). Unfortunately, in Xenopus eggs, a system in which antisense inhibition would be extremely useful for developmental studies, endonucleases are also very abundant. Oligonucleotides blocked at both ends are degraded rapidly after microinjection (Doug Melton, Harvard University). Degradation of the targeted message is observed at high concentrations of the oligonucleotide (Xenopus eggs are very rich in RNase $\mathrm{H}$ ), but the injection of the required amount of oligonucleotide is toxic to the embryo.

Why not use antisense RNA in the Xenopus system? Although RNA : RNA duplexes do not form substrates for RNase $\mathrm{H}$, hybridization of a long antisense RNA sequence to the $5^{\prime}$-end of a mRNA may block translation directly (Melton 1985). Unfortunately, antisense RNAs do not work well in Xenopus eggs either. The discovery of a helix unwinding activity in Xenopus eggs provided a cogent explanation for this result (Bass and Weintraub 1987; Regbagliati and Melton 1987), but the problem may be more complex. Harold Weintraub (Fred Hutchinson Cancer Research Center) presented evidence that this factor not only unwinds RNA : RNA duplexes but also modifies the RNA strands in such a manner that they 
are unable to rehybridize. It would seem unlikely that such an extensively modified mRNA would still be translatable. If that supposition proves to be true, the unwinding activity would not account for the unmasking of maternal mRNAs during differentiation, as initially suggested, or the failure of antisense RNAs to arrest translation of targeted mRNAs in Xenopus eggs. Whatever their function, such unwinding factors appear to be widely distributed. Richard Wagner and Kazuko Nishikura (Wistar Institute) reported the presence of a similar unwinding activity in HeLa cells and in five other mammalian cell lines. In mouse 3T3 fibroblasts, the activity is expressed in a cell cycle-specific manner. The level of expression is very low in cells arrested in quiescence and it increases sharply upon induction of proliferation with fetal calf serum.

Modification of RNA by the unwinding factors found in Xenopus and other systems interferes with its detection on Northern blots, and may lead to the erroneous conclusion that the targeted message has been degraded. To determine if degradation actually has occurred, the message must be probed for outside of the region complementary to the antisense RNA.

Recently, inhibition of expression of the $\lambda \mathrm{cII}$ gene by endogenous antisense RNA has been shown to be dependent on cleavage of the mRNA by RNase III (Krinke and Wulff 1987). RNase III cleaves both the sense and the antisense strands in the RNA : RNA duplex. The target of the antisense RNA is at the $3^{\prime}$ end of the message. The transposase gene of the insertion element IS10 is also regulated post-transcriptionally by antisense RNA (Nancy Kleckner, Harvard University). In this case, the target sequence lies at the $5^{\prime}$ end of the message. In wild-type cells, the RNA : RNA duplex formed is cleaved rapidly by RNase III and the mRNA is degraded (Robert Simons, University of California, Los Angeles). Cleavage of the message, however, is not essential for inhibition. In an RNase III-deficient mutant, cleavage of the message does not occur, and there is relatively little effect on the extent of inhibition. Hybridization of the antisense RNA to the $5^{\prime}$ end of the message alone is sufficient to block translation.

Inhibition of the expression of the transposase gene by antisense RNA was shown to be dependent on integration host factor (IHF), a histone-like protein of E. coli (Simons). IHF was first identified by its requirement for chromosomal integration of $\lambda$. Here it appears to function like the Rom protein to promote hybridization of sense and antisense RNAs (Tomizawa and Sam 1984).

RNase III-like activity is also found in eukaryotic cells, but its role in the blockade of gene expression by antisense RNA remains uncertain. It is clear, however, that degradation of the mRNA is not a prerequisite for inhibition, even for antisense RNAs that hybridize at the $3^{\prime}$ end of the message. Antisense RNA complementary to the $3^{\prime}$ untranslated region of the creatine kinase $B$ gene very efficiently inhibits expression of the protein in U937 cells, without effect on the level of the mature mRNA within the cytoplasm (Edward Holmes, Duke University Medical Center). The mRNA does not appear to have been modified by an unwinding factor because it remains capable of hybridization over the target sequence. Perhaps in this case, hybridization blocks normal translation termination.

Many other examples demonstrating the utility of antisense DNA and RNA were presented at this meeting. Space permits the mention of only a few. All trypanosome mRNAs contain a common leader sequence of 35 nucleotides at their $5^{\prime}$ end (Walder et al. 1986; Cornelissen et al. 1986). Jean-Jacques Toulme (INSERM) reported that a nonanucleotide complementary to a portion of this sequence, and containing an acridine derivative attached to the $3^{\prime}$ end of the molecule, killed $T$. brucei cells in culture. Although high concentrations of the oligonucleotide were required $(80 \mu \mathrm{M})$, the effect was shown to be sequence specific. The acridine group is essential for activity. Whether this is due to facilitated uptake or decreased degradation of the oligonucleotide, or to increased stability of the DNA : RNA duplex due to intercalation of the dye, is unknown.

Randy Moon /University of Washington School of Medicine) described the use of antisense RNA to inhibit the expression of the cytoskeletal protein 4.1 during embryonic development in Xenopus laevis. As in the studies described above, microinjection of the antisense RNA into Xenopus eggs was ineffective. However, microinjection of a plasmid bearing the antisense sequence flanked by the Moloney sarcoma virus long terminal repeat to provide for a high level of expression proved to be very successful. By the gastrula stage, antisense transcripts became detectable in poly(A) ${ }^{+}$RNA, and, thereafter, cytoplasmic levels of the endogenous 4.1 transcript decreased dramatically. Kinetic studies suggest that expression of the antisense RNA resulted in degradation of preexisting 4.1 message as well as newly synthesized mRNA. The plasmid persisted for at least 14 days. The antisense RNA continued to be expressed and blocked synthesis of 4.1 in the tadpole retina.

Microinjection of antisense RNA was used to study the regulation of the expression of tissue plasminogen activator ( $t$-PA) during maturation of the mouse oocyte in studies reported by Sidney Strickland (State University of New York, Stony Brook). In the primary oocyte, t-PA mRNA has a very short poly(A) tail (less than 40 residues) and is not translated. Upon transition to the secondary oocyte, a long poly(A) stretch is added to the $3^{\prime}$ end of the mRNA ( $\sim 500$ residues), and the message is translated and also becomes unstable. Microinjection of an antisense RNA complementary to the $3^{\prime}$-noncoding region resulted in cleavage of the mRNA at the site of hybridization, presumably by an RNase III-like activity. This blocked addition of the poly $(A)$ tail, the induction of translation, and the decrease in message stability that normally occur upon maturation to the secondary oocyte. The addition of the poly(A) sequence may provide a signal for the latter two events. The loss of t-PA expression does not produce any obvious phenotype. The oocyte appears to mature normally in vitro and can still be fertilized.

Marcelo Jacobs-Lorena (Case Western Reserve School of Medicine) used antisense RNA to inhibit the synthesis of the Drosophila ribosomal protein rpAl. An an- 
tisense copy of the gene driven by the heat shock hsp70 promoter was introduced into the germ line of the fly by $P$ element-mediated transfection. Expression of the antisense gene severely disrupted oogenesis, but had no demonstrable effect later in development. Females expressing the gene produced small, unfertile eggs. Inhibition of the synthesis of rpAl appeared to interfere with the normal transfer of constituents to the egg from surrounding nurse cells.

Antisense RNA was used by Susan Lindquist (University of Chicago) to explore the function of heat shock proteins in Drosophila. Tissue culture cells were transformed stably with antisense genes that were placed under control of the hsp70 promoter, as in the studies described by Jacobs-Lorena. Expression of an antisense gene directed against hsp 26 markedly inhibited induction of the protein during heat shock, without effect on the closely related genes for hsp28, hsp23, and hsp22. The targeted mRNA was either degraded or rendered unhybridizable, producing no effect on either the synthesis of other heat shock proteins or in the recovery of cells from heat shock; this is consistent with the observation that in yeast deletion of hsp26 produces no apparent phenotype (Petko and Lindquist 1986). Expression of an antisense gene against hsp 70 achieved a reduction in the synthesis of the protein during heat shock to $25 \%$ of the normal level. Overexpression of other heat shock proteins occurred, and the synthesis of hsp70 extended for a longer period during recovery, indicating that hsp70 may be required for attenuation of the response. After exposure to $36.5^{\circ} \mathrm{C}$ for $45 \mathrm{~min}$, the rate of restoration of normal protein synthesis was the same as in control cells; however, with more severe heat shock, the rate of recovery of the antisense cell line was delayed.

The function of developmentally regulated Dictyostelium genes was studied by Rick Firtel (University of California, San Diego/ using antisense inhibition experiments. A family of vectors was constructed into which antisense genes could be cloned downstream of promoters for genes that are expressed at specific stages of Dictyostelium development. Inhibition of the expression of discoidin I, a fibronectin homolog, with antisense RNA encoded on an integrating vector blocked the formation of aggregation streams as in known discoidin I-minus mutants. Plasmids containing an antisense ras gene yielded no transformants, suggesting that ras is as essential for Dictyostelium as it is for yeast. Firtel also proposed an intriguing procedure for shotgun antisense mutagenesis using autonomously replicating plasmids that are stably maintained in Dictyostelium as cloning vectors for cDNA or genomic libraries. Fragments are cloned downstream of a developmentally regulated promoter, and transformants are then screened for developmental mutant phenotypes.

Successful examples of the use of antisense DNA and RNA are accumulating steadily, as is our understanding of their mechanism of action. The results reported here, hopefully, are harbingers of a more general applicability of the method.

\section{Joseph Walder}

Department of Biochemistry

University of Iowa

Iowa City, Iowa USA

\section{References}

Bass, B.L. and H. Weintraub. 1987. A developmentally regulated activity that unwinds RNA Duplexes. Cell 48: 607-613.

Cornelissen, A.W.C.A., M.P. Verspieren, J. Toulme, B.W. Swinkels, and P. Borst. 1986. The common 5' terminal sequence on trypanosome mRNAs: a target for anti-messenger oligodeoxynucleotides. Nucleic Acids Res. 14: 5606-5614.

Holt, J.T., R.L. Redner, and A.W. Nienhuis. 1988. An oligomer complementary to c-myc mRNA inhibits proliferation of HL-60 promyelocytic cells and induces differentiation. Mol. Cell. Biol. 8: 963-973.

Krinke, L. and D.L. Wulff. 1987. OOP RNA, produced from multi copy plasmids, inhibits lambda cII gene expression through an RNase III-dependent mechanism. Genes Dev. 1: $1005-1013$.

Lawson, T.G., B.K. Ray, J.T. Dodds, J.A. Grifo, R.D. Abramson, W.C. Merrick, D.F. Betsch, H.L. Weith, and R.E. Thach. 1986. Influence of $5^{\prime}$ proximal secondary structure on the translation efficiency of Eukaryotic mRNAs and on their interaction with initiation factors. J. Bio. Chem. 260: 1397913989.

Melton D.A. 1985. Injected anti-sense RNAs specifically block messenger RNA translation in vivo. Proc. Natl. Acad. Sci. 82: $144-148$.

Petko, L. and S. Lindquist. 1986. Hsp26 is not required for growth at high temperatures, nor for thermotolerance, spore development, or germination. Cell 45: 885-894.

Rebagliati, M.R. and D.A. Melton. 1987. Antisense RNA injections in fertilized frog eggs reveal an RNA duplex unwinding activity. Cell 48: 599-605.

Tomizawa, J. and T. Som. 1984. Control of ColE1 plasmid replication: enhancement of binding of RNA 1 to the primer transcript by the Rom protein. Cell 38: $871-878$.

Walder, J.A., P.S. Eder, D.M. Engman, S.T. Brentano, R.Y. Walder, D.S. Knutzon, D.M. Dorfman, and J.E. Donelson. 1986. The 35-nucleotide spliced leader sequence is common to all trypanosome messenger RNAs. Science 233: 569-571.

Wickstrom, E.L., T.A. Bacon, A. Gonzalez, D.L. Freeman, G.H. Lyman, and E. Wickstrom. 1988. Human promyelocytic leukemia HL-60 cell proliferation and c-myc protein expression are inhibited by an antisense pentadecadeoxynucleotide targeted against c-myc mRNA. Proc. Natl. Acad. Sci. 85: $1028-1032$.

Zamecnik, P.C. and M.L. Stephenson. 1978. Inhibition of Rous sarcoma virus replication and cell transformation by a specific oligodeoxynucleotide. Proc. Nat1. Acad. Sci. 75: 280284. 


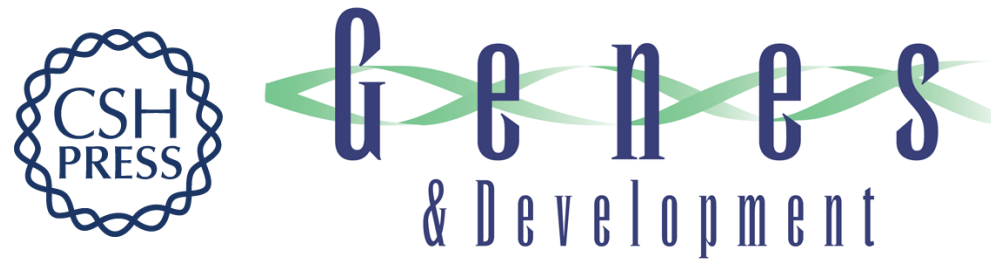

\section{Antisense DNA and RNA: progress and prospects.}

J Walder

Genes Dev. 1988, 2:

Access the most recent version at doi:10.1101/gad.2.5.502 $\begin{array}{ll}\text { References } & \text { This article cites } 12 \text { articles, } 6 \text { of which can be accessed free at: } \\ \text { http://genesdev.cshlp.org/content/2/5/502.full.html\#ref-list-1 }\end{array}$

License

Email Alerting

Receive free email alerts when new articles cite this article - sign up in the box at the top Service right corner of the article or click here.

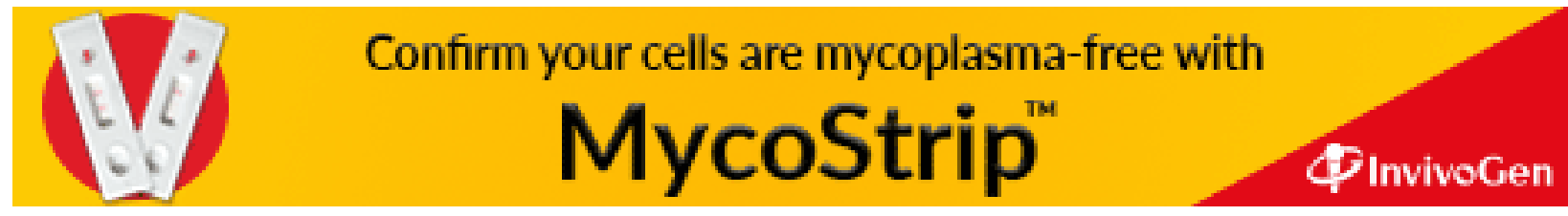

